\title{
On the spectra of a new duplication based corona of graphs
}

\author{
Renny P. Varghese $^{1}$ and D. Susha ${ }^{2}$ \\ ${ }^{1}$ Department of Mathematics, Catholicate College \\ Pathanamthitta, Kerala 689 645, India \\ e-mail: rennypv1@gmail.com \\ ${ }^{2}$ Department of Mathematics, Catholicate College \\ Pathanamthitta, Kerala 689 645, India \\ e-mail: sushad70@gmail.com
}

Received: 10 November $2019 \quad$ Revised: 28 January $2021 \quad$ Accepted: 1 February 2021

\begin{abstract}
In this paper we introduce a new corona-type product of graphs namely duplication corresponding corona. Here we mainly determine the adjacency, Laplacian and signless Laplacian spectra of the new graph product. In addition to that we find out the incidence energy, the number of spanning trees, Kirchhoff index and Laplacian-energy-like invariant of the new graph. Also we discuss some new classes of cospectral graphs.
\end{abstract}

Keywords: Spectrum, Corona, Duplication graph, Incidence energy, Spanning tree, Kirchhoff index, Laplacian-energy-like invariant.

2010 Mathematics Subject Classification: 05C50, 05 C76.

\section{Introduction}

The graphs considered in this paper have no loops and parallel edges and are undirected. Let $G$ be an arbitrary graph with vertex set $V(G)=\left\{v_{1}, v_{2}, \ldots, v_{n}\right\}$. Let $A(G)$ be the adjacency matrix of $G$. It is an $n \times n$ symmetric matrix, $A(G)=\left(a_{i j}\right)_{n \times n}$, where $a_{i j}=1$ if $v_{i}$ and $v_{j}$ are connected by an edge in $G$, and 0 elsewhere.

Let the degree of $v_{i}$ in $G$ be $d_{i}$ (number of vertices adjacent to $v_{i}$ ) and the diagonal degree matrix of $G$ be $D(G)=\operatorname{diag}\left(d_{1}, d_{2}, \ldots, d_{n}\right)$. Laplacian matrix and signless Laplacian matrix are 
defined as $L(G)=D(G)-A(G)$ and $Q(G)=D(G)+A(G)$, respectively. For more details see $[2,4,7,14]$. The characteristic polynomial of the graph $G$ is defined as $f_{G}(A: x)=\operatorname{det}\left(x I_{n}-A\right)$, where $I_{n}$ is the identity matrix of order $n$. The eigenvalues of $G$ are the roots of $f_{G}(A: x)=0$. They are denoted by $\lambda_{1} \geq \lambda_{2} \geq \cdots \geq \lambda_{n}$ and usually called adjacency spectrum or $A$-spectrum of $G$. Similarly the eigenvalues of $L(G)$ and $Q(G)$ are denoted by $0=\mu_{1} \leq \mu_{2} \leq \cdots \leq \mu_{n}$ and $\nu_{1} \geq \nu_{2} \geq \cdots \geq \nu_{n}$. They are called the Laplacian and signless Laplacian spectrum (or $L$-spectrum and $Q$-spectrum respectively) of $G$. The eigenvalues of $A(G), L(G)$ and $Q(G)$ are real numbers since the matrices are real and symmetric. Cospectral graphs are those graphs with the same spectrum.

Let $G$ be a graph with vertex set $V(G)=\left\{v_{1}, v_{2}, \ldots, v_{n}\right\}$. Let $U(G)=\left\{x_{1}, x_{2}, \ldots, x_{n}\right\}$ be another set. Draw $x_{i}$ adjacent to all the vertices in $\mathcal{N}\left(v_{i}\right)$, the neighborhood set of $v_{i}$, in $G$ for each $i$ and delete the edges of $G$ only. The graph thus obtained is called the duplication graph [15] of $G$ and we denote it as $\mathcal{D G}$. Let $G_{1}$ and $G_{2}$ be two graphs with $n_{1}$ and $n_{2}$ vertices. The corona of $G_{1}$ and $G_{2}$ is the graph obtained by taking one copy of $G_{1}$ and $n_{1}$ copies of $G_{2}$ and joining $i$-th vertex of $G_{1}$ to every vertices in the $i$-th copy of $G_{2}$. Frucht and Harary in [5] introduced this concept and their spectrum by Cvetković et. al [3]. Gopalapillai in [6] introduced neighborhood corona of graphs and calculated the corresponding spectrum. In [17,19] Varghese and Susha defined some new join and corona-type graphs based on duplication graph of an arbitrary graph. In $[10,16,18]$ new product related to corona of graph are studied. Motivated from these works, here we define a new corona-type graph namely, duplication corresponding corona and determine its adjacency, Laplacian and signless Laplacian spectrum.

The paper is organized as follows. In Section 2 we state some necessary preliminaries. In Section 3 we define duplication corresponding corona and find their adjacency, Laplacian and signless Laplacian spectrum. Also we discuss some concepts like the number of spanning trees, the Kirchhoff index and the incidence energy of the new graph. Also we introduce some new classes of cospectral graphs using this new product.

\section{Preliminaries}

Lemma 2.1 ([2]). Let $M=\left[\begin{array}{cc}M_{1} & M_{2} \\ M_{2} & M_{1}\end{array}\right]$ be a symmetric block matrix of order $2 \times 2$. Then the eigenvalues of $M$ are those of $M_{1}+M_{2}$ together with $M_{1}-M_{2}$.

Proposition 2.2 ([2]). Let $P_{0}, P_{1}, P_{2}$ and $P_{3}$ be matrices of order $n_{1} \times n_{1}, n_{1} \times n_{2}, n_{2} \times n_{1}, n_{2} \times n_{2}$ respectively. Then

$$
\operatorname{det}\left[\begin{array}{ll}
P_{0} & P_{1} \\
P_{2} & P_{3}
\end{array}\right]= \begin{cases}\operatorname{det}\left(P_{0}\right) \operatorname{det}\left(P_{3}-P_{2} P_{0}^{-1} P_{1}\right), & \text { if } P_{0} \text { is invertible } \\
\operatorname{det}\left(P_{3}\right) \operatorname{det}\left(P_{0}-P_{1} P_{3}^{-1} P_{2}\right), & \text { if } P_{3} \text { is invertible }\end{cases}
$$

Definition 2.3 ([13]). Let $A$ be the adjacency matrix of a graph $G$ with $n$ vertices. The determinant, $\operatorname{det}(x I-A)=f_{G}(A: x) \neq 0$, is invertible being the characteristic matrix of $A$. The $A$-coronal, $\chi_{A}(x)$, of $G$ is defined to be the sum of the entries of the matrix $(x I-A)^{-1}$. We denote this as

$$
\chi_{A}(x)=\mathbf{j}_{n}^{T}(x I-A)^{-1} \mathbf{j}_{n},
$$


where $\mathbf{j}_{n}$ is a $n \times 1$ column vector with all entries equal to 1 .

Let $A=\left(a_{i j}\right)$ and $B$ be matrices. Then the Kronecker product, $A \otimes B$, of $A$ and $B$ is defined as the partition matrix $\left(a_{i j} B\right)$. For details see [2].

- $(A \otimes B)^{T}=A^{T} \otimes B^{T}$, where $A$ and $B$ are of appropriate order;

- $(A+B) \otimes C=A \otimes C+B \otimes C ;$

- $(A \otimes B)(C \otimes D)=A C \otimes B D$ whenever the product $A C$ and $B D$ exist;

- $(A \otimes B)^{-1}=A^{-1} \otimes B^{-1}$ for the non-singular matrix $A$ and $B$;

- $\operatorname{det}(A \otimes B)=(\operatorname{det} A)^{p}(\operatorname{det} B)^{n}$ where $A$ and $B$ are $n \times n$ and $p \times p$ matrices.

\section{Duplication corresponding corona of graphs}

The following definition describes a new graph corona-type product based on the duplication graph of a graph.

Definition 3.1. Let $G_{1}$ and $G_{2}$ be two vertex disjoint graphs with $n_{1}$ and $n_{2}$ vertices, respectively. Let $V\left(G_{1}\right) \cup U\left(G_{1}\right)$ where, $V\left(G_{1}\right)=\left\{v_{1}, v_{2}, \ldots, v_{n_{1}}\right\}$ and $U\left(G_{1}\right)=\left\{x_{1}, x_{2}, \ldots, x_{n_{1}}\right\}$ be vertex set of $\mathcal{D} \mathcal{G}_{1}$, the duplication graph of $G_{1}$. The vertex $x_{i}$ is a duplication of $v_{i}$ for each $i=1,2, \ldots n_{1}$. Duplication corresponding corona of $G_{1}$ and $G_{2}$, denoted by $G_{1} * G_{2}$ and is the graph obtained from $\mathcal{D} \mathcal{G}_{1}$ and $n_{1}$ copies of $G_{2}$ by making $x_{i}$ and $v_{i}$ adjacent to every vertices in the $i$-th copy of $G_{2}$ for $i=1,2, \ldots, n_{1}$.

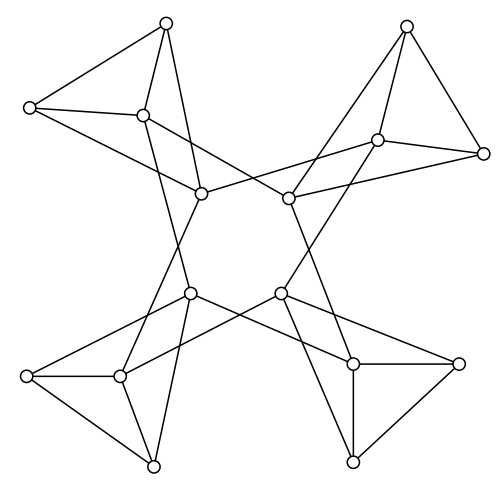

Figure 1. Duplication corresponding corona $C_{4} \stackrel{*}{-} K_{2}$

Theorem 3.2. Let $G_{i}$ be two graphs with spectrum $\lambda_{i 1} \geq \lambda_{i 2} \geq \cdots \geq \lambda_{i n_{i}}$ for $i=1,2$. Then the characteristic polynomial of $G_{1} \star G_{2}$ is

$$
f_{G_{1} * G_{2}}(A: x)=\prod_{j=1}^{n_{2}}\left(x-\lambda_{2 j}\right)^{n_{1}} \prod_{i=1}^{n_{1}}\left(x+\lambda_{1 i}\right) \prod_{i=1}^{n_{1}}\left(x-2 \chi_{A_{2}}(x)-\lambda_{1 i}\right) .
$$


Proof. Let $G_{1}$ and $G_{2}$ be two graphs with $n_{1}$ and $n_{2}$ vertices, respectively. Let the vertex set of $G_{1}$ be $V\left(G_{1}\right)=\left\{v_{1}, v_{2}, \ldots, v_{n_{1}}\right\}$ and $U\left(G_{1}\right)=\left\{x_{1}, x_{2}, \ldots, x_{n_{1}}\right\}$ be a set of vertices corresponding to $V\left(G_{1}\right)$. The vertex in the $i$-th copy of $G_{2}$ is $\left\{u_{1}^{i}, u_{2}^{i}, \ldots, u_{n_{2}}^{i}\right\}$ and let $W_{j}=\left\{u_{j}^{1}, u_{j}^{2}, \ldots, u_{j}^{n_{2}}\right\}$ for $j=1,2, \ldots, n_{1}$. Then $V\left(G_{1}\right) \cup U\left(G_{1}\right) \cup\left\{W_{1} \cup W_{2} \cup \ldots \cup W_{n_{1}}\right\}$ is a vertex partition of $G_{1} \stackrel{*}{*} G_{2}$ with $n_{1}\left(n_{2}+2\right)$ vertices.

By this vertex partitioning, the block form of the adjacency matrix of $G_{1} * G_{2}$ is

$$
A\left(G_{1} \stackrel{*}{*} G_{2}\right)=\left[\begin{array}{ccc}
0 & A_{1} & \mathbf{j}_{n_{2}}^{T} \otimes I_{n_{1}} \\
A_{1} & 0_{n_{1} \times n_{1}} & \mathbf{j}_{n_{2}}^{T} \otimes I_{n_{1}} \\
\mathbf{j}_{n_{2}} \otimes I_{n_{1}} & \mathbf{j}_{n_{2}} \otimes I_{n_{1}} & A_{2} \otimes I_{n_{1}}
\end{array}\right],
$$

where $A_{1}$ and $A_{2}$ are the adjacency matrices of $G_{1}$ and $G_{2}$, respectively. $\mathbf{j}_{n_{2}}$ is a $n_{2} \times 1$ column vector with all entries equal to 1 and $I_{n_{1}}$ is an identity matrix of order $n_{1}$.

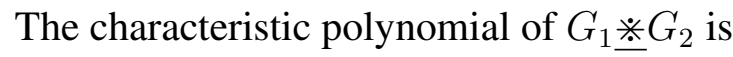

$$
\begin{aligned}
& f_{G_{1} \stackrel{*}{*} G_{2}}(A: x)=\left|\begin{array}{ccc}
x I_{n_{1}} & -A_{1} & -\mathbf{j}_{n_{2}}^{T} \otimes I_{n_{1}} \\
-A_{1} & x I_{n_{1}} & -\mathbf{j}_{n_{2}}^{T} \otimes I_{n_{1}} \\
-\mathbf{j}_{n_{2}} \otimes I_{n_{1}} & -\mathbf{j}_{n_{2}} \otimes I_{n_{1}} & \left(x I_{n_{2}}-A_{2}\right) \otimes I_{n_{1}}
\end{array}\right|, \\
& =\operatorname{det}\left(\left(x I_{n_{2}}-A_{2}\right) \otimes I_{n_{2}}\right) \operatorname{det}(S) \text {, } \\
& \text { where, } S=\left[\begin{array}{cc}
x I_{n_{1}} & -A_{1} \\
-A_{1} & x I_{n_{1}}
\end{array}\right] \\
& -\left[\begin{array}{l}
-\mathbf{j}_{n_{2}}^{T} \otimes I_{n_{1}} \\
-\mathbf{j}_{n_{2}}^{T} \otimes I_{n_{1}}
\end{array}\right]\left(\left(x I_{n_{2}}-A_{2}\right) \otimes I_{n_{1}}\right)^{-1}\left[\begin{array}{ll}
-\mathbf{j}_{n_{2}} \otimes I_{n_{1}} & -\mathbf{j}_{n_{2}} \otimes I_{n_{1}}
\end{array}\right] .
\end{aligned}
$$

By using the property of Kronecker product we can substantiate that

$$
\left(\mathbf{j}_{n_{2}}^{T} \otimes I_{n_{1}}\right)\left(\left(x I_{n_{2}}-A_{2}\right)^{-1} \otimes I_{n_{1}}\right)\left(\mathbf{j}_{n_{2}} \otimes I_{n_{1}}\right)=\chi_{A_{2}}(x) I_{n_{1}}
$$

Therefore,

$$
\begin{aligned}
S & =\left[\begin{array}{cc}
x I_{n_{1}} & -A_{1} \\
-A_{1} & x I_{n_{1}}
\end{array}\right]-\left[\begin{array}{ll}
\chi_{A_{2}}(x) I_{n_{1}} & \chi_{A_{2}}(x) I_{n_{1}} \\
\chi_{A_{2}}(x) I_{n_{1}} & \chi_{A_{2}}(x) I_{n_{1}}
\end{array}\right], \\
& =\left[\begin{array}{cc}
x I_{n_{1}}-\chi_{A_{2}}(x) I_{n_{1}} & -A_{1}-\chi_{A_{2}}(x) I_{n_{1}} \\
-A_{1}-\chi_{A_{2}}(x) I_{n_{1}} & x I_{n_{1}}-\chi_{A_{2}}(x) I_{n_{1}}
\end{array}\right] .
\end{aligned}
$$

This implies that, $\operatorname{det}(S)=\operatorname{det}(x I-M)$, where

$$
M=\left[\begin{array}{cc}
\chi_{A_{2}}(x) I_{n_{1}} & A_{1}+\chi_{A_{2}}(x) I_{n_{1}} \\
A_{1}+\chi_{A_{2}}(x) I_{n_{1}} & \chi_{A_{2}}(x) I_{n_{1}}
\end{array}\right] .
$$

By Lemma 2.1 the eigenvalues of $M$ are those of $A_{1}+2 \chi_{A_{2}}(x) I_{n_{1}}$ and $-A_{1}$, i.e., the roots of $\operatorname{det}\left(x I_{n_{1}}-2 \chi_{A_{2}}(x) I_{n_{1}}-A_{1}\right)=0$ and $\operatorname{det}\left(x I_{n_{1}}+A_{1}\right)=0$.

Also

$$
\begin{aligned}
\operatorname{det}\left(\left(x I_{n_{2}}-A_{2}\right) \otimes I_{n_{1}}\right) & =\left(\operatorname{det}\left(x I_{n_{2}}-A_{2}\right)\right)^{n_{1}}\left(\operatorname{det}\left(I_{n_{1}}\right)\right)^{n_{2}} \\
& =\prod_{j=1}^{n_{2}}\left(x-\lambda_{2 j}\right)^{n_{1}} .
\end{aligned}
$$


Hence,

$$
f_{G_{1} * G_{2}}(A: x)=\prod_{j=1}^{n_{2}}\left(x-\lambda_{2 j}\right)^{n_{1}} \prod_{i=1}^{n_{1}}\left(x+\lambda_{1 i}\right) \prod_{i=1}^{n_{1}}\left(x-2 \chi_{A_{2}}(x)-\lambda_{1 i}\right) .
$$

Corollary 3.3. For $i=1,2$, let $G_{i}$ be two graphs with $n_{i}$ vertices with spectrum $\lambda_{i 1} \geq \lambda_{i 2} \geq$

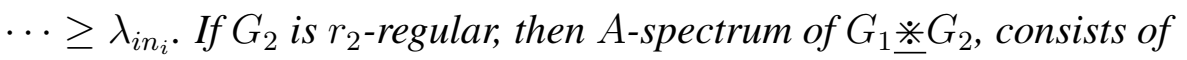

1. $\lambda_{2 j}$, repeated $n_{1}$ times for $j=2,3, \ldots, n_{2}$;

2. $-\lambda_{1 i}$, for $i=1,2, \ldots, n_{1}$;

3. $\frac{\left(r_{2}+\lambda_{1 i}\right) \pm \sqrt{\left(r_{2}-\lambda_{1 i}\right)^{2}+8 n_{2}}}{2}$, for $i=1,2, \ldots, n_{1}$.

Proof. Since $G_{2}$ is $r_{2}$-regular, from Equation 1 we get, $\chi_{A_{2}}(x)=\frac{n_{2}}{x-r_{2}}$.

$$
\begin{aligned}
x-2 \chi_{A_{2}}(x)-\lambda_{1 i} & =x-2 \frac{n_{2}}{x-r_{2}}-\lambda_{1 i} \\
& =\frac{x^{2}-x r_{2}-2 n_{2}-x \lambda_{1 i}+r_{2} \lambda_{1 i}}{x-r_{2}} \\
& =\frac{x^{2}-\left(\lambda_{1 i}+r_{2}\right) x+r_{2} \lambda_{1 i}-2 n_{2}}{x-r_{2}} .
\end{aligned}
$$

Hence from Theorem 3.2 and use the fact $\lambda_{21}=r_{2}$, we get

$$
f_{G_{1} \underset{*}{*} G_{2}}(A: x)=\prod_{j=2}^{n_{2}}\left(x-\lambda_{2 j}\right)^{n_{1}} \prod_{i=1}^{n_{1}}\left(x+\lambda_{1 i}\right) \prod_{i=1}^{n_{1}}\left(x^{2}-\left(\lambda_{1 i}+r_{2}\right) x+r_{2} \lambda_{1 i}-2 n_{2}\right) .
$$

This completes the proof of the corollary.

Corollary 3.4. Let $G_{1}$ be an arbitrary graph with $n_{1}$ vertices and spectrum $\lambda_{11} \geq \lambda_{12} \geq \cdots \geq$ $\lambda_{1 n_{1}}$. If $G_{2}=\overline{K_{n_{2}}}$ (totally disconnected graph on $n_{2}$ vertices), then A-spectrum of $G_{1} \triangleq G_{2}$ consists of,

1. 0 , repeated $n_{1}\left(n_{2}-1\right)$ times;

2. $-\lambda_{1 i}$, for $i=1,2, \ldots, n_{1}$;

3. $\frac{\lambda_{1 i} \pm \sqrt{\lambda_{1 i}^{2}+8 n_{2}}}{2}$, for $i=1,2, \ldots, n_{1}$.

Proof. Since $G_{2}$ is $\overline{K_{n_{2}}}, \chi_{A_{2}}(x)=\frac{n_{2}}{x}$ and the $\lambda_{2 j}=0$ for $j=1,2, \ldots, n_{2}$.

Using Theorem 3.2 and Corollary 3.3 we get the characteristic polynomial as,

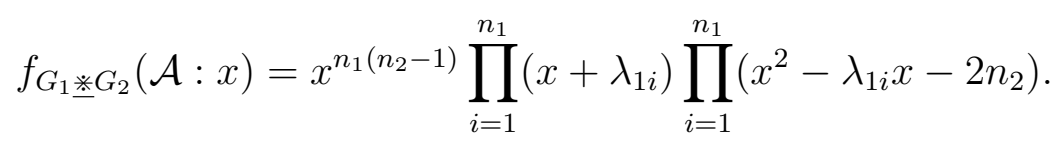

This completes the proof of the corollary. 
Theorem 3.5. Let $G_{1}$ be an $r_{1}$-regular graph on $n_{1}$ vertices and $G_{2}$ be an arbitrary graph on $n_{2}$ vertices with Laplacian spectrum $0=\mu_{j 1} \leq \mu_{j 2} \leq \cdots \leq \mu_{j n_{j}}$ for $j=1,2$. Then $L$ - spectrum of $G_{1} * G_{2}$ consists of,

(i) 0 ;

(ii) $n_{2}+2$;

(iii) $2+\mu_{2 i}$, repeated $n_{1}$ times for $i=2,3, \ldots, n_{2}$;

(iii) $n_{2}+2 r_{1}-\mu_{1 i}$, for $i=1,2, \ldots, n_{1}$;

(iv) Two roots of the equation,

$$
x^{2}-\left(n_{2}+\mu_{1 i}+2\right) x+2 \mu_{1 i}=0, i=2,3, \ldots, n_{1} .
$$

Proof. Let $G_{1}$ be an $r_{1}$-regular graph with $n_{1}$ vertices and $G_{2}$ be an arbitrary graph with $n_{2}$ vertices. Let $V\left(G_{1}\right)=\left\{v_{1}, v_{2}, \ldots, v_{n_{1}}\right\}$ be the vertex set of $G_{1}$ and $U\left(G_{1}\right)=\left\{x_{1}, x_{2}, \ldots, x_{n_{1}}\right\}$ be the set of vertices corresponding to $V\left(G_{1}\right)$. The vertex in the $i$-th copy of $G_{2}$ is $\left\{u_{1}^{i}, u_{2}^{i}, \ldots, u_{n_{2}}^{i}\right\}$. The degree of the vertices of $G_{1} * G_{2}$ are $d_{G_{1} * G_{2}}\left(v_{i}\right)=d_{G_{1} * G_{2}}\left(x_{i}\right)=n_{2}+r_{1}, i=1,2, \ldots, n_{1}$ and $d_{G_{1} * G_{2}}\left(u_{j}^{i}\right)=d_{G_{2}}\left(u_{j}\right)+2$, for $i=1,2, \ldots, n_{1}$ and $j=1,2, \ldots, n_{2}$.

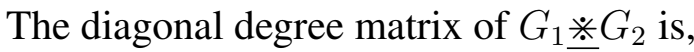

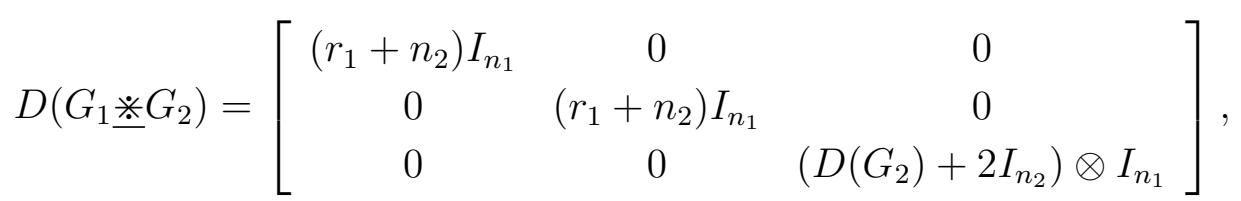

where $D\left(G_{2}\right)$ is the diagonal degree matrix of the graph $G_{2}$.

$$
\begin{aligned}
\left(D\left(G_{2}\right)+2 I_{n_{2}}\right) \otimes I_{n_{1}}-A_{2} \otimes I_{n_{1}} & =\left(D\left(G_{2}\right)+2 I_{n_{2}}-A_{2}\right) \otimes I_{n_{1}} \\
& =\left(L_{2}+2 I_{n_{2}}\right) \otimes I_{n_{1}} .
\end{aligned}
$$

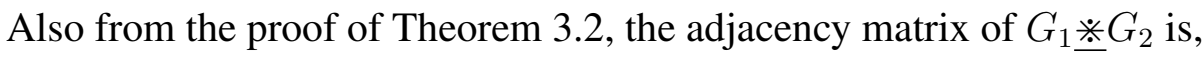

$$
A=\left[\begin{array}{ccc}
0 & A_{1} & \mathbf{j}_{n_{2}}^{T} \otimes I_{n_{1}} \\
A_{1} & 0_{n_{1} \times n_{1}} & \mathbf{j}_{n_{2}}^{T} \otimes I_{n_{1}} \\
\mathbf{j}_{n_{2}} \otimes I_{n_{1}} & \mathbf{j}_{n_{2}}^{T} \otimes I_{n_{1}} & A_{2} \otimes I_{n_{1}}
\end{array}\right]
$$

where $A_{1}$ and $A_{2}$ are the adjacency matrices of $G_{1}$ and $G_{2}$, respectively. The Laplacian matrix of $G_{1} \stackrel{*}{*} G_{2}$ is,

$$
\begin{aligned}
L & =D-A \\
& =\left[\begin{array}{ccc}
\left(r_{1}+n_{2}\right) I_{n_{1}} & -A_{1} & -\mathbf{j}_{n_{2}}^{T} \otimes I_{n_{1}} \\
-A_{1} & \left(r_{1}+n_{2}\right) I_{n_{1}} & -\mathbf{j}_{n_{2}}^{T} \otimes I_{n_{1}} \\
-\mathbf{j}_{n_{2}} \otimes I_{n_{1}} & -\mathbf{j}_{n_{2}} \otimes I_{n_{1}} & \left(L_{2}+2 I_{n_{2}}\right) \otimes I_{n_{1}}
\end{array}\right],
\end{aligned}
$$

where $L_{2}$ is the Laplacian matrix of the graph $G_{2}$.

Here we use the properties of Kronecker product and can easily proved that

$$
\left(\mathbf{j}_{n_{2}}^{T} \otimes I_{n_{1}}\right)\left(\left((x-2) I_{n_{2}}-L_{2}\right)^{-1} \otimes I_{n_{1}}\right)\left(\mathbf{j}_{n_{2}} \otimes I_{n_{1}}\right)=\chi_{L_{2}}(x-2) I_{n_{1}} .
$$


The Laplacian characteristic polynomial of $G_{1} * G_{2}$ is,

$$
\begin{aligned}
& f_{G_{1} * G_{2}}(L: x)=\left|\begin{array}{ccc}
\left(x-r_{1}-n_{2}\right) I_{n_{1}} & A_{1} & \mathbf{j}_{n_{2}}^{T} \otimes I_{n_{1}} \\
A_{1} & \left(x-r_{1}-n_{2}\right) I_{n_{1}} & \mathbf{j}_{n_{2}}^{T} \otimes I_{n_{1}} \\
\mathbf{j}_{n_{2}} \otimes I_{n_{1}} & \mathbf{j}_{n_{2}} \otimes I_{n_{1}} & \left((x-2) I_{n_{2}}-L_{2}\right) \otimes I_{n_{1}}
\end{array}\right| \\
& =\operatorname{det}\left(\left((x-2) I_{n_{2}}-L_{2}\right) \otimes I_{n_{1}}\right) \operatorname{det}(S) \text {, } \\
& \text { where, } S=\left[\begin{array}{cc}
\left(x-r_{1}-n_{2}\right) I_{n_{1}} & A_{1} \\
A_{1} & \left(x-r_{1}-n_{2}\right) I_{n_{1}}
\end{array}\right]
\end{aligned}
$$

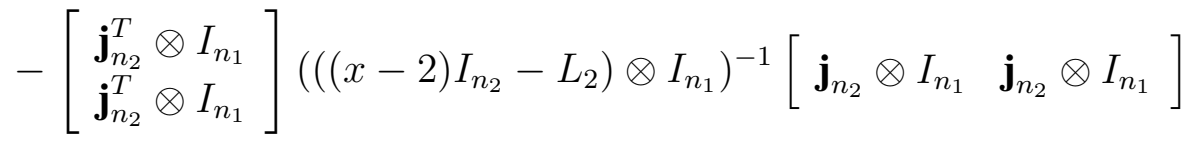

$$
\begin{aligned}
& =\left[\begin{array}{cc}
\left(x-r_{1}-n_{2}\right) I_{n_{1}} & A_{1} \\
A_{1} & \left(x-r_{1}-n_{2}\right) I_{n_{1}}
\end{array}\right] \\
& -\left[\begin{array}{cc}
\chi_{L_{2}}(x-2) I_{n_{1}} & \chi_{L_{2}}(x-2) I_{n_{1}} \\
\chi_{L_{2}}(x-2) I_{n_{1}} & \chi_{L_{2}}(x-2) I_{n_{1}}
\end{array}\right] \\
& =\left[\begin{array}{cc}
\left(x-r_{1}-n_{2}-\chi_{L_{2}}(x-2)\right) I_{n_{1}} & A_{1}-\chi_{L_{2}}(x-2) I_{n_{1}} \\
A_{1}-\chi_{L_{2}}(x-2) I_{n_{1}} & \left(x-r_{1}-n_{2}-\chi_{L_{2}}(x-2)\right) I_{n_{1}}
\end{array}\right] \text {. }
\end{aligned}
$$

Hence, $\operatorname{det}(S)=\operatorname{det}(x I-M)$, where,

$$
M=\left[\begin{array}{cc}
\left(r_{1}+n_{2}+\chi_{L_{2}}(x-2)\right) I_{n_{1}} & -A_{1}+\chi_{L_{2}}(x-2) I_{n_{1}} \\
-A_{1}+\chi_{L_{2}}(x-2) I_{n_{1}} & \left(r_{1}+n_{2}+\chi_{L_{2}}(x-2)\right) I_{n_{1}}
\end{array}\right] .
$$

By Lemma 2.1 the eigenvalues of $M$ are those of $\left(r_{1}+n_{2}+\chi_{L_{2}}(x-2)\right) I_{n_{1}}-A_{1}+\chi_{L_{2}}(x-2) I_{n_{1}}$ together with $\left(r_{1}+n_{2}+\chi_{L_{2}}(x-2)\right) I_{n_{1}}+A_{1}-\chi_{L_{2}}(x-2) I_{n_{1}}$, i.e., those of $\left(r_{1}+n_{2}+2 \chi_{L_{2}}(x-2)\right) I_{n_{1}}-A_{1}$ together with $\left(n_{2}+r_{1}\right) I_{n_{1}}+A_{1}$.

We use the fact that $L=D-A$, so, for a $r_{1}$-regular graph we have $L=r_{1} I_{n_{1}}-A_{1}$ and $\mu_{1 i}=r_{1}-\lambda_{1 i}$ for $i=1,2, \ldots, n$.

Therefore the eigenvalues of $M$ are those of $\left(n_{2}+2 \chi_{L_{2}}(x-2)\right) I_{n_{1}}+L_{1}$ together with $\left(n_{2}+2 r_{1}\right) I_{n_{1}}-L_{1}$.

$$
\begin{aligned}
\operatorname{det}\left(\left((x-2) I_{n_{2}}-L_{2}\right) \otimes I_{n_{1}}\right) & =\operatorname{det}\left((x-2) I_{n_{2}}-L_{2}\right)^{n_{1}}\left(\operatorname{det}\left(I_{n_{1}}\right)\right)^{n_{2}} \\
& =\prod_{j=1}^{n_{2}}\left(x-2-\mu_{2 j}\right)^{n_{1}} .
\end{aligned}
$$

Hence,

$$
f_{G_{1} \text { } G_{2}}(L: x)=\prod_{j=1}^{n_{2}}\left(x-2-\mu_{2 j}\right)^{n_{1}} \prod_{i=1}^{n_{1}}\left(x-2 r_{1}-n_{2}+\mu_{1 i}\right) \prod_{i=1}^{n_{1}}\left(x-n_{2}-2 \chi_{L_{2}}(x-2)-\mu_{1 i}\right) .
$$

Using equation (1) we have,

$$
\chi_{L_{2}}(x-2)=\frac{n_{2}}{x-2} .
$$




$$
\begin{aligned}
x-n_{2}-2 \chi_{L_{2}}(x-2)-\mu_{1 i} & =x-n_{2}-\frac{2 n_{2}}{x-2}-\mu_{1 i} \\
& =\frac{x^{2}-\left(n_{2}+2+\mu_{1 i}\right) x+2 \mu_{1 i}}{x-2} \\
\prod_{i=1}^{n_{1}}\left(x-n_{2}-2 \chi_{L_{2}}(x-2)-\mu_{1 i}\right) & =\frac{x\left(x-n_{2}-2\right)}{(x-2)^{n_{1}}} \prod_{i=2}^{n_{1}}\left(x^{2}-\left(n_{2}+2+\mu_{1 i}\right) x+2 \mu_{1 i}\right)
\end{aligned}
$$

Also, since $\mu_{11}=\mu_{21}=0$, the characteristic polynomial becomes

$$
\begin{aligned}
f_{G_{1} \text { } G_{2}}(L: x)= & x\left(x-n_{2}-2\right) \prod_{j=2}^{n_{2}}\left(x-2-\mu_{2 j}\right)^{n_{1}} \prod_{i=1}^{n_{1}}\left(x-n_{2}-2 r_{1}+\mu_{1 i}\right) \\
& \prod_{i=2}^{n_{1}}\left(x^{2}-\left(n_{2}+\mu_{1 i}+2\right) x+2 \mu_{1 i}\right) .
\end{aligned}
$$

Hence the theorem is proved.

Remark 3.6. Spanning tree of a graph is a subgraph of it which is also a tree. The number of spanning trees of a graph $G$ is denoted by $t(G)$. If $G$ is a connected graph with $n$ vertices and the Laplacian spectrum $0=\mu_{1}(G) \leq \mu_{2}(G) \leq \cdots \leq \mu_{n}(G)$, then [11] the number of spanning trees

$$
t(G)=\frac{\mu_{2}(G) \mu_{3}(G) \cdots \mu_{n}(G)}{n} .
$$

Corollary 3.7. Let $G_{1}$ be an $r_{1}$-regular graph on $n_{1}$ vertices and $G_{2}$ be an arbitrary graph on $n_{2}$

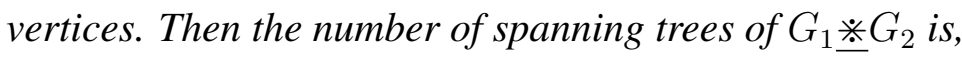

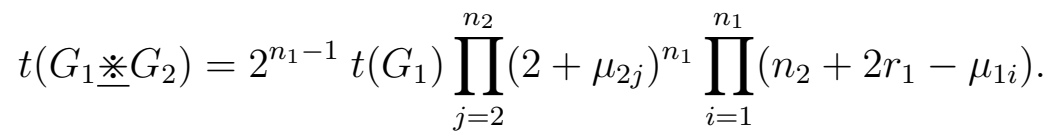

Proof. The proof follows from Theorem 3.5 and equation (2).

Remark 3.8. Klein and Randić in [9] introduced a new notion named resistance distance based on electric resistance in a network corresponding to a graph, in which the resistance distance between any two adjacent vertices is $1 \mathrm{ohm}$. The electric resistance is calculated by means of the Kirchhoff laws called Kirchhoff index.

For a graph $G$ with $n$ vertices, where $n \geq 2$, and the Laplacian spectrum $0=\mu_{1}(G)$ $\leq \mu_{2}(G) \leq \cdots \leq \mu_{n}(G)$, then the Kirchhoff index, $K f(G)$, is defined as

$$
K f(G)=n \sum_{i=2}^{n} \frac{1}{\mu_{i}} .
$$

Corollary 3.9. Let $G_{1}$ be an $r_{1}$-regular graph on $n_{1}$ vertices and $G_{2}$ be an arbitrary graph on $n_{2}$

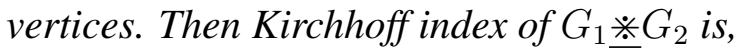

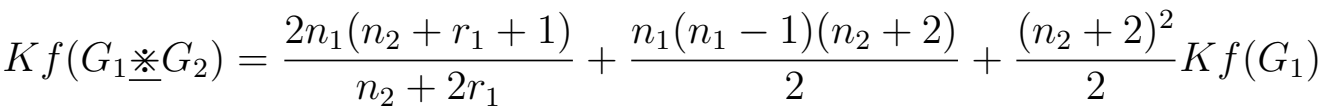

$$
\begin{aligned}
& +n_{1}\left(n_{2}+2\right)\left[\sum_{j=2}^{n_{2}} \frac{n_{1}}{2+\mu_{2 j}}+\sum_{i=2}^{n_{1}} \frac{1}{n_{2}+2 r_{1}-\mu_{1 i}}\right] .
\end{aligned}
$$


Proof. Let $y_{i 1}$ and $y_{i 2}$ be the roots of the equation $x^{2}-\left(n_{2}+\mu_{1 i}+2\right) x+2 \mu_{1 i}=0, i=2,3, \ldots, n_{1}$.

$$
\begin{aligned}
\frac{1}{y_{i 1}}+\frac{1}{y_{i 2}} & =\frac{y_{i 1}+y_{i 2}}{y_{i 1} y_{i 2}} \\
& =\frac{n_{2}+2+\mu_{1 i}}{2 \mu_{1 i}} \\
& =\frac{n_{2}+2}{2 \mu_{1 i}}+\frac{1}{2} \\
\sum_{i=2}^{n_{1}}\left(\frac{1}{y_{i 1}}+\frac{1}{y_{i 2}}\right) & =\frac{n_{2}+2}{2 n_{1}} K f\left(G_{1}\right)+\frac{n_{1}-1}{2} .
\end{aligned}
$$

The remaining proof follows from Theorem 3.5 and equation (3).

J. Liu and B. Liu defined a new Laplacian graph invariant in [12], Laplacian-energy-like invariant (LEL).

Let $G$ be a graph with $n$ vertices and the Laplacian spectrum $0=\mu_{1}(G) \leq \mu_{2}(G) \cdots \leq$ $\mu_{n}(G)$. LEL of $G$ is defined as,

$$
L E L(G)=\sum_{i=2}^{n} \sqrt{\mu_{i}}
$$

Corollary 3.10. Let $G_{1}$ be an $r_{1}$-regular graph on $n_{1}$ vertices and $G_{2}$ be an arbitrary graph on $n_{2}$ vertices with Laplacian spectrum $\left\{0=\mu_{1}, \mu_{2}, \ldots, \mu_{n}\right\}$. Then,

$$
\begin{aligned}
\operatorname{LEL}\left(G_{1} \stackrel{*}{*} G_{2}\right) & =\sqrt{n_{2}+2}+n_{1} \sum_{j=2}^{n_{2}} \sqrt{2+\mu_{2 j}}+\sum_{i=1}^{n_{1}} \sqrt{n_{2}+2 r_{1}-\mu_{1 i}} \\
& +\sum_{i=2}^{n_{1}}\left[\left(n_{2}+\mu_{1 i}+2\right)+2 \sqrt{2 \mu_{1 i}}\right]^{1 / 2} .
\end{aligned}
$$

Proof. The proof follows from Theorem 3.5, equation (4) and the identity

$$
(\sqrt{x}+\sqrt{y})^{2}=(x+y)+2 \sqrt{x y} .
$$

Corollary 3.11. For two $L$-cospectral regular graphs $G_{1}$ and $G_{2}$ and an arbitrary graph $H$, the graphs $G_{1} * H$ and $G_{2} * H$ are L-cospectral. Also if $G$ is a regular graph and $H_{1}$ and $H_{2}$ are $L$-cospectral graphs, then $G \star H_{1}$ and $G * H_{2}$ are L-cospectral.

Theorem 3.12. Let $G_{1}$ be an $r_{1}$-regular graph on $n_{1}$ vertices and $G_{2}$ be an arbitrary graph on $n_{2}$ vertices with signless Laplacian spectrum $\nu_{i 1} \geq \nu_{i 2} \geq \cdots \geq \nu_{i n_{i}}$ for $i=1,2$, respectively. Then

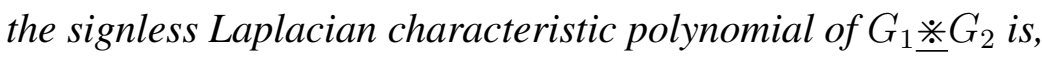

$$
f_{G_{1} \text { } G_{2}}(Q: x)=\prod_{j=1}^{n_{2}}\left(x-2-\nu_{2 j}\right)^{n_{1}} \prod_{i=1}^{n_{1}}\left(x-n_{2}-2 r_{1}+\nu_{1 i}\right) \prod_{i=1}^{n_{1}}\left(x-n_{2}-2 \chi_{Q_{2}}(x-2)-\nu_{1 i}\right) .
$$

Proof. As like the notations defined in Theorem 3.5, we can define the signless Laplacian matrix

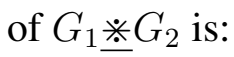




$$
\begin{aligned}
Q & =\left[\begin{array}{ccc}
\left(r_{1}+n_{2}\right) I_{n_{1}} & A_{1} & \mathbf{j}_{n_{2}}^{T} \otimes I_{n_{1}} \\
A_{1} & \left(r_{1}+n_{2}\right) I_{n_{1}} & \mathbf{j}_{n_{2}}^{T} \otimes I_{n_{1}} \\
\mathbf{j}_{n_{2}} \otimes I_{n_{1}} & \mathbf{j}_{n_{2}} \otimes I_{n_{1}} & \left(2 I_{n_{2}}+Q_{2}\right) \otimes I_{n_{1}}
\end{array}\right] . \\
f_{G_{1} * G_{2}}(Q: x) & =\left|\begin{array}{ccc}
\left(x-r_{1}-n_{2}\right) I_{n_{1}} & -A_{1} & -\mathbf{j}_{n_{2}}^{T} \otimes I_{n_{1}} \\
-A_{1} & \left(x-r_{1}-n_{2}\right) I_{n_{1}} & -\mathbf{j}_{n_{2}}^{T} \otimes I_{n_{1}} \\
-\mathbf{j}_{n_{2}} \otimes I_{n_{1}} & -\mathbf{j}_{n_{2}} \otimes I_{n_{1}} & \left((x-2) I_{n_{2}}-Q_{2}\right) \otimes I_{n_{1}}
\end{array}\right|, \\
& =\operatorname{det}\left((x-2) I_{n_{2}}-Q_{2}\right) \otimes I_{n_{1}} \operatorname{det}(S),
\end{aligned}
$$

where $Q_{2}$ is the signless Laplacian matrix of $G_{2}$.

As like in Theorem 3.5 proved above,

$$
S=\left[\begin{array}{cc}
\left(x-r_{1}-n_{2}-\chi_{Q_{2}}(x-2)\right) I_{n_{1}} & -A_{1}-\chi_{Q_{2}}(x-2) I_{n_{1}} \\
-A_{1}-\chi_{Q_{2}}(x-2) I_{n_{1}} & \left(x-r_{1}-n_{2}-\chi_{Q_{2}}(x-2)\right) I_{n_{1}}
\end{array}\right] .
$$

Hence $\operatorname{det} S=\operatorname{det}(x I-M)$, where,

$$
M=\left[\begin{array}{cc}
\left(r_{1}+n_{2}+\chi_{Q_{2}}(x-2)\right) I_{n_{1}} & A_{1}+\chi_{Q_{2}}(x-2) I_{n_{1}} \\
A_{1}+\chi_{Q_{2}}(x-2) I_{n_{1}} & \left(r_{1}+n_{2}+\chi_{Q_{2}}(x-1)\right) I_{n_{1}}
\end{array}\right] .
$$

By Lemma 2.1 the eigenvalues of $M$ are those of $\left(r_{1}+n_{2}+2 \chi_{Q_{2}}(x-2)\right) I_{n_{1}}+A_{1}$ together with $\left(r_{1}+n_{2}\right) I_{n_{1}}-A_{1}$.

We use the fact that $Q=D+A$, since $G_{1}$ is an $r_{1}$-regular graph we have $Q=r_{1} I+A_{1}$ and then, $\nu_{1 i}=r_{1}+\lambda_{1 i}$ for $i=1,2, \ldots, n$.

Therefore, the eigenvalues of $M$ are those of $\left(n_{2}+2 \chi_{Q_{2}}(x-2)\right) I_{n_{1}}+Q_{1}$ together with $\left(n_{2}+2 r_{1}\right) I_{n_{1}}-Q_{1}$.

Also,

$$
\begin{aligned}
\operatorname{det}\left(\left((x-2) I_{n_{2}}-Q_{2}\right) \otimes I_{n_{1}}\right) & =\operatorname{det}\left((x-2) I_{n_{2}}-Q_{2}\right)^{n_{1}}\left(\operatorname{det}\left(I_{n_{1}}\right)\right)^{n_{2}} \\
& =\prod_{j=1}^{n_{2}}\left(x-2-\nu_{2 j}\right)^{n_{1}} .
\end{aligned}
$$

Hence,

$$
f_{G_{1} \nVdash G_{2}}(Q: x)=\prod_{j=1}^{n_{2}}\left(x-2-\nu_{2 j}\right)^{n_{1}} \prod_{i=1}^{n_{1}}\left(x-n_{2}-2 r_{1}+\nu_{1 i}\right) \prod_{i=1}^{n_{1}}\left(x-n_{2}-2 \chi_{Q_{2}}(x-2)-\nu_{1 i}\right) .
$$

This completes the proof.

Corollary 3.13. Let $G_{i}$ be an $r_{i}$-regular graph on $n_{i}$ vertices $i=1,2$ with signless Laplacian

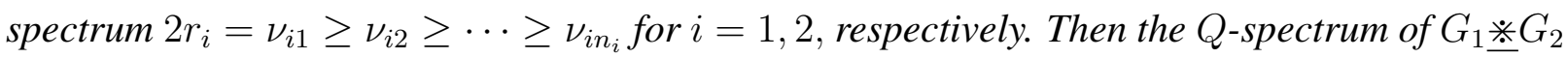
consists of:

(i) $\nu_{2 i}+2$, repeats $n_{1}$ times for $i=2,3, \ldots, n_{2}$;

(ii) $n_{2}+2 r_{1}-\nu_{1 i}$ for $i=1,2, \ldots, n_{1}$; 
(iii) two roots of the equation

$$
x^{2}-\left(n_{2}+2 r_{2}+\nu_{1 i}+2\right) x+2\left(n_{2} r_{2}+\nu_{1 i}+r_{2} \nu_{1 i}\right)=0 \text { for } i=2,3, \ldots, n_{2} .
$$

Proof. Since $G_{2}$ is $r_{2}$-regular, $\nu_{21}=2 r_{2}$. Using equation (1) we have,

$$
\chi_{Q_{2}}(x-2)=\frac{n_{2}}{x-2-2 r_{2}} .
$$

Therefore,

$$
\begin{aligned}
f_{G_{1} \text { } G_{2}}(Q: x)= & \prod_{j=2}^{n_{2}}\left(x-2-\nu_{2 j}\right)^{n_{1}} \prod_{i=1}^{n_{1}}\left(x-2 r_{1}-n_{2}+\nu_{1 i}\right) \\
& \prod_{i=1}^{n_{1}}\left(x^{2}-\left(2+2 r_{2}+n_{2}+\nu_{1 i}\right) x-2\left(n_{2} r_{2}+\nu_{1 i}+r_{2} \nu_{1 i}\right)\right) .
\end{aligned}
$$

Corollary 3.14. For two $Q$-cospectral regular graphs $G_{1}$ and $G_{2}$ and an arbitrary graph $H$, the graphs $G_{1} * H$ and $G_{2} * H$ are $Q$-cospectral. Also if $G$ is a regular graph and $H_{1}$ and

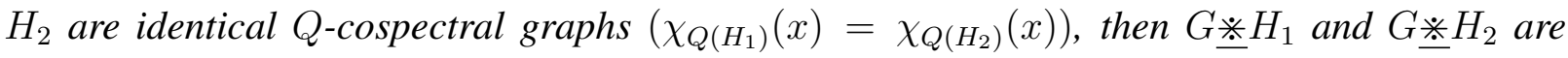
Q-cospectral.

Let $A$ be any real $n \times m$ matrix with transpose $A^{T}$. The square roots of the eigenvalues of $A A^{T}$ are called the singular values of the matrix $A$. The incidence matrix [1] of $G$ is the $0-1$ matrix $R=\left(r_{i j}\right)$ with rows indexed by vertices and columns by edges where $r_{i j}=1$ when the vertex $v_{i}$ is an end point of the edge $e_{j}$ and 0 otherwise.

Definition 3.15. [8] Let $\rho_{1}, \rho_{2}, \ldots, \rho_{n}$ be the singular values of the incidence matrix $R$ of a graph $G$. The incidence energy of $G$ is defined as $\mathcal{I E}(G)=\sum_{i=1}^{n} \rho_{i}$.

It is clear that $\mathcal{I E}(G) \geq 0$ and the equality holds if and only if $G$ is totally disconnected. We have, $R R^{T}=Q$ [1] and let $\nu_{1} \geq \nu_{2} \geq \cdots \geq \nu_{n}$ be the signless Laplace spectrum of $G$. In [8] Jooyandeh et. al. defined the incidence energy as

$$
\mathcal{I E}(G)=\sum_{i=1}^{n} \sqrt{\nu_{i}} .
$$

Corollary 3.16. For $i=1,2$ let $G_{i}$ be an $r_{i}$-regular graph on $n_{i}$ vertices with signless Laplacian

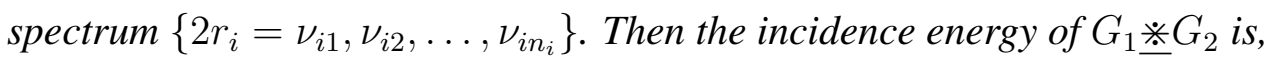

$$
\begin{aligned}
\mathcal{I} \mathcal{E}\left(G_{1} \stackrel{*}{*} G_{2}\right)= & n_{1} \sum_{j=2}^{n_{2}} \sqrt{2+\nu_{2 j}}+\sum_{i=1}^{n_{1}} \sqrt{n_{2}+2 r_{1}-\nu_{1 i}} \\
& +\sum_{i=1}^{n_{1}}\left[\left(n_{2}+2 r_{2}+\mu_{1 i}+2\right)+2 \sqrt{2\left(n_{2} r_{2}+\nu_{1 i}+r_{2} \nu_{1 i}\right)}\right]^{1 / 2} .
\end{aligned}
$$

Proof. The proof follows the identity $(\sqrt{x}+\sqrt{y})^{2}=(x+y)+2 \sqrt{x y}$ and using Corollary 3.13 and Equation (5). 
Let $x_{i 1}$ and $x_{i 2}$ be the roots of the equation $x^{2}-\left(n_{2}+2 r_{2}+\nu_{1 i}+2\right) x+2\left(n_{2} r_{2}+\nu_{1 i}+r_{2} \nu_{1 i}\right)=0$.

$$
\begin{aligned}
\left(\sqrt{x_{i 1}}+\sqrt{x_{i 2}}\right)^{2} & =\left(x_{i 1}+x_{i 2}\right)+2 \sqrt{x_{i 1} x_{i 2}} \\
& =\left(n_{2}+2 r_{2}+\mu_{1 i}+2\right)+2 \sqrt{2\left(n_{2} r_{2}+\nu_{1 i}+r_{2} \nu_{1 i}\right)} \\
\sqrt{x_{i 1}}+\sqrt{x_{i 2}} & =\left[\left(n_{2}+2 r_{2}+\mu_{1 i}+2\right)+2 \sqrt{2\left(n_{2} r_{2}+\nu_{1 i}+r_{2} \nu_{1 i}\right)}\right]^{1 / 2} .
\end{aligned}
$$

Then the corollary follows.

\section{Acknowledgements}

The authors are thankful to the reviewer for their comments and suggestions for improving the quality of this paper. Also the author thankful to the University Grants Commission of Government of India for providing fellowship under the FDP in the XII th plan.

\section{References}

[1] Brouwer, A. E., \& Haemers, W. H. (2012). Spectra of Graphs, Springer.

[2] Cvetković, D. M., Doob, M., \& Sachs, H. (1995). Spectra of Graphs, Theory and Applications, Third edition, Johann Ambrosius Barth, Heidelberg.

[3] Cvetković, D. M., Gutman, I. \& Simic, S. K. (1978). On self pseudo-inverse graphs, Univ. Beograd Publ. Elektrotehn. Fak., Ser. Mat. Fiz., 602-633, 111-117.

[4] Cvetković, D. M., \& Simic, S. K. (2009). Towards a spectral theory of graphs based on the signless Laplacian. Publications de l'Institut Mathematique, Nouvelle series, 85(99), 19-33.

[5] Frucht, H., \& Harary, F. (1970). On the corona of two graphs. Aequationes Mathematicae, $4,322-325$.

[6] Gopalapillai, I. (2011). The spectrum of neighborhood corona of graphs. Kragujevac Journal of Mathematics, 35, 493-500.

[7] Grone, R., Merris, R., \& Sunder, V. S. (1990). The Laplacian spectrum of a graph. SIAM Journal on Matrix Analysis and Applications, 11, 218-238.

[8] Jooyandeh, M., Kiania, D., \& Mirzakhaha, M. (2009). Incidence energy of a graph. MATCH Communications in Mathematical and in Computer Chemistry, 62, 561-572.

[9] Klein, D. J., \& Randić, M. (1993). Resistance distance. Journal of Mathematical Chemistry, $12,81-95$.

[10] Kumar, K. R., \& Varghese, R. P. (2017). Spectrum of $(k, r)$-regular hypergraphs. International Journal of Mathematical Combinatorics, 2, 52-59. 
[11] Li, J., Shiu, W. C., \& Chang, A. (2010). The number of spanning tree of a graph. Applied Mathematics Letters, 23, 286-290.

[12] Liu, J., \& Liu, B. (2008). A Laplacian-energy-like invariant of a graph. MATCH Communications in Mathematical and in Computer Chemistry, 59, 397-413.

[13] McLeman, C., \& McNicholas, E. (2011). Spectra of coronae. Linear Algebra and its Applications, 435, 998-1007.

[14] Merris, R. (1994). Laplacian matrices of graphs: A survey. Linear Algebra and its Applications, 197, 143-176.

[15] Sampathkumar, E. (1973). On duplicate graphs. Journal of the Indian Mathematical Society, 37, 285-293.

[16] Sarkar, P., De, N., \& Pal, A. (2020). Zagreb indices of double join and double corona of graphs based on the total graph, International Journal of Applied and Computational Mathematics, 6, Art. No. 73.

[17] Varghese, R. P., \& Susha, D. (2017). Spectrum of some new product of graphs and its applications. Global Journal of Pure and Applied Mathematics, 13(9), 4493-4504.

[18] Varghese, R. P. \& Susha, D. (2017). The spectrum of two new corona of graphs and its applications. International Journal of Mathematics And Its Applications, 5(4-C), 395-406.

[19] Varghese, R. P., \& Susha, D. (2020). On the normalized Laplacian spectrum of some graphs. Kragujevac Journal of Mathematics, 44(3), 431-442. 